\title{
Advances in the genomics of ticks and tick-borne pathogens
}

\section{Frans Jongejan ${ }^{1,2}$, Vishvanath Nene ${ }^{3}$, Jose de la Fuente, ${ }^{4,5}$, Arnab Pain ${ }^{6}$ and Peter Willadsen ${ }^{7}$}

\author{
${ }^{1}$ Utrecht Centre for Tick-Borne Diseases (UCTD), Department of Infectious Diseases and Immunology, Faculty of Veterinary \\ Medicine, Utrecht University, Yalelaan 1, 3584 CL, Utrecht, The Netherlands \\ ${ }^{2}$ Department of Veterinary Tropical Diseases, Faculty of Veterinary Science, University of Pretoria, Private Bag X04, 0110, \\ Onderstepoort, South Africa \\ ${ }^{3}$ The Institute for Genomic Research, 9712 Medical Centre Drive, Rockville, MD 20850, USA \\ ${ }^{4}$ Department of Veterinary Pathobiology, Center for Veterinary Health Sciences, Oklahoma State University, Stillwater, OK 74078, \\ USA \\ ${ }^{5}$ Instituto de Investigación en Recursos Cinegéticos IREC (CSIC-UCLM-JCCM), Ronda de Toledo s/n, 13071, Ciudad Real, Spain \\ ${ }^{6}$ The Pathogen Sequencing Unit, The Wellcome Trust Sanger Institute, Genome Campus, Hinxton, Cambridge CB10 1SA, UK \\ ${ }^{7}$ CSIRO Livestock Industries, Queensland Bioscience Precinct, 306 Carmody Road, St Lucia, QLD 4067, Australia
}

Ticks and the diseases for which they are vectors engage in complex interactions with their mammalian hosts. These interactions involve the developmental processes of tick and pathogen, and interplay between the defensive responses and counter responses of host, tick and pathogen. Understanding these interactions has long been an intractable problem, but progress is now being made thanks to the flood of genomic information on host, tick and pathogen, and the attendant, novel experimental tools that have been generated. Each advance reveals new levels of complexity, but there are encouraging signs that genomics is leading to novel means of parasite control.

\section{Ticks: parasites and disease vectors}

Ticks, as blood-feeding ectoparasites, affect their hosts both directly and as vectors of viral, bacterial and protozoal diseases. Their impact as disease vectors on human wellbeing is second only to that of mosquitoes, and their effect on livestock, wildlife and domestic animals is immeasurably greater [1]. An important source of information on ticks and tick-borne pathogens is described in Box 1.

The tick's mode of feeding means it must maintain intimate contact with the host in the face of host defensive responses for a protracted time. In the tick-host-pathogen triangle, we therefore have the cut and thrust of parasite attack, host response and parasite counter-response, and the interactions between transmitted pathogen and the vector tick. This results in a highly complex biological system that is barely understood. Although there are common elements in the strategies adopted by both ticks and tick-borne pathogens, there is also great diversity in the details and in the molecules that facilitate these interactions. This is illustrated in the accompanying poster (Bell-Sakyi and Jongejan, this issue).

Corresponding author: Willadsen, P. (Peter.Willadsen@csiro.au).

Available online 26 July 2007.
Genomics is having an impact on this field of research in three ways: first, through the generation of new resources, including genome information and experimental techniques; second, through the application of these new resources, coupled with traditional biological research techniques, to the acquisition of new insights; and, third, through the development of practical new interventions in the tick-host-pathogen triangle.

\section{Tick-borne pathogens: genome sequences and} comparative genomics

Striking achievements have been made in the acquisition of genome sequence information. Web-based resources are summarized in Box 2.

Although it is early days, the interpretation of genome sequences is leading to new biological understanding. Examples include antigenic variation in Anaplasma marginale, which has long been the subject of active research. Completion of the $A$. marginale genome and the genomes of related organisms has enabled a comparative approach to this question [2]. Analysis of genome sequences from organisms as diverse as A. marginale, Borrelia hermsii and Trypanosoma brucei suggests convergent evolution to common mechanisms of antigenic variation and hence immune evasion (Palmer and Brayton, this issue). Comparative genomics analysis of several pathogenic and nonpathogenic organisms of the order Rickettsiales, including the tick-borne pathogens A. marginale, Anaplasma phagocytophilum, Ehrlichia chaffeensis and Ehrlichia ruminantium, shows some common pathogen features, for instance, decreased ability to synthesize amino acids. However, differences also emerge relating to host pathology, the presence or absence of transovarial transmission and adaptation to a haematophagous vector [3], as well as the expansion of families of immunodominant outer membrane proteins, facilitating antigenic variation. The generation of multiple $E$. ruminantium genome sequences has enabled a third series of comparative approaches [4,5] 


\section{Box 1. Tick-host-pathogen database (THPbase)}

The THPbase database (www.icttd.nl), facilitated by the Integrated Consortium on Ticks and Tick-borne Diseases (ICTTD-3), is a searchable database of all known tick species, tick-borne pathogens and associated hosts. It was originally created as Ticksbase, a peerreviewed global species database of Ixodidae incorporated into the Species 2000 Catalogue of Life program (www.sp2000.org), which has the aim of indexing the world's known species [43].

The database includes taxonomic information for 889 valid tick species, 580 common names and over 3000 synonyms, distribution data and a bibliography. In addition, a section on vector competence includes 808 tick-pathogen relationships. An effort to extend THPbase with genomics information is ongoing.

(Frutos et al., this issue) to understanding the active process of genome evolution.

Our first insight into the genome of a spirochaete came from sequencing the Lyme disease pathogen Borrelia burgdorferi (strain B31) [6]. The genome is unusual. It includes a linear chromosome $\sim 1 \mathrm{Mb}$ in size, and at least 17 linear and circular plasmids that collectively represent more than half of the chromosome in size [6]. Borrelias appear to have lost the full gene repertoire for cellular biosynthesis and have a significantly reduced metabolic capability. More recently, the availability of the genome sequences of three species of Borrelia (i.e. B. burgdorferri sensu stricto, $B$. garinii and B. afzelii) has enhanced our understanding of the subtle differences between the genomes of these Lyme borreliosis-associated pathogens $[7,8]$. The major phenotypic differences between these species could be attributed to variability in the presence of plasmid sequences and to genes under positive selection $[7,8]$. In this issue, Hovius et al. review novel insights into how $B$. burgdorferi interacts with particular tick salivary proteins (Salp15) to facilitate its transmission to the mammalian host [9].

\section{Box 2. Web resources for the genomics of ticks and tick-borne pathogens}

The National Center for Biotechnology Information (NCBI; http:// www.ncbi.nlm.nih.gov/), European Molecular Biology Laboratory (EMBL; http://www.ebi.ac.uk/embl/) and DNA Data Bank of Japan (DDBJ; http://www.ddbj.nig.ac.jp/) house a large number of DNA and protein sequences, and are usually the best source of publicly available data submitted to these data banks. At the NCBI, the 'Taxonomy browser' is a good starting point for surveying the different types of sequences available for any species of interest.

In the Genome Reviews database (http://www.ebi.ac.uk/GenomeReviews/) at the European Bioinformatics Institute (EBI), almost all of the completely sequenced tick-borne pathogen (prokaryotic and eukaryotic) genomes that are deposited in EMBL are available in an up-todate and standardised format. The Institute for Genomic Research (TIGR) Comprehensive Microbial Resources site (http://cmr.tigr.org/ tigr-scripts/CMR/CmrHomePage.cgi) contains more than 300 prokaryotic genomes.

Genome sequence data on species of particular significance to those interested in ticks and tick-borne and related pathogens can also be accessed via the Internet as listed in Table 1.

Table 1. Genome sequence data on species of particular significance

\begin{tabular}{|c|c|}
\hline Database or host site & Species of interest \\
\hline The Gene Index project, The Dana Faber Cancer Institute & Amblyomma variegatum \\
\hline \multirow[t]{3}{*}{ http://compbio.dfci.harvard.edu/tgi/tgipage.html } & Rhipicephalus appendiculatus \\
\hline & Boophilus microplus \\
\hline & Ixodes scapularis \\
\hline Genome Center, University Oklahoma & Amblyomma americanum \\
\hline \multicolumn{2}{|l|}{ http://www.genome.ou.edu/tick.html } \\
\hline Ixodes scapularis Genome Project, Purdue University & Ixodes scapularis \\
\hline \multicolumn{2}{|l|}{ http://www.entm.purdue.edu/igp/ } \\
\hline \multicolumn{2}{|l|}{ VectorBase, NIAID } \\
\hline \multicolumn{2}{|l|}{ http://www.vectorbase.org/index.php } \\
\hline GeneDB, The Wellcome Trust Sanger Institute & Theileria annulata \\
\hline http://www.genedb.org/ & Plasmodium species \\
\hline Apicomplexan Database Resources & Plasmodium species \\
\hline \multirow[t]{3}{*}{ http://apidb.org/apidb/ } & Toxoplasma gondii \\
\hline & Cryptosporidium species \\
\hline & Theileria species \\
\hline University of Pretoria and Onderstepoort Veterinary Institute & Ehrlichia ruminantium \\
\hline \multicolumn{2}{|l|}{ http://www.bi.up.ac.za/Ehrlichia_ruminantium/ } \\
\hline PathoSystems Resource Integration Center & Rickettsia species \\
\hline \multicolumn{2}{|l|}{ https://patric.vbi.vt.edu/ } \\
\hline \multicolumn{2}{|l|}{ Washington State University } \\
\hline & Babesia bovis (BLAST-based queries) \\
\hline \multicolumn{2}{|l|}{$\begin{array}{l}\text { http://www.vetmed.wsu.edu/research_vmp/babesia-bovis } \\
\text { (http://genomics.vetmed.wsu.edu/blast/blast.html) }\end{array}$} \\
\hline The Wellcome Trust Sanger Institute & Babesia bigemina (BLAST-based queries) \\
\hline \multicolumn{2}{|l|}{ http://www.sanger.ac.uk/Projects/B_bigemina } \\
\hline NCBI virus genome database & Genomes and related information on all tick-borne viruses \\
\hline \multicolumn{2}{|l|}{ http://www.ncbi.nlm.nih.gov/genomes/static/vis.html } \\
\hline $\begin{array}{l}\text { Washington State University } \\
\text { http://www.vetmed.wsu.edu/research_vmp/anagenome }\end{array}$ & Anaplasma marginale \\
\hline Ohio State University & Anaplasma phagocytophilum \\
\hline http://riki-lb1.vet.ohio-state.edu/ehrlichia/ & Ehrlichia chaffeensis \\
\hline
\end{tabular}


Several species of Theileria and Babesia of the phylum Apicomplexa are major tick-borne protozoan parasites responsible for life-threatening diseases of animals. The genome sequences of two closely related Theileria species (Theileria parva and Theileria annulata) have been described [10,11]. Multilevel genome comparison between Theileria spp. and Plasmodium (malaria) parasites shows the nuclear genomes of both Theileria species to be highly compact $\left[\sim 8.3 \times 10^{6}\right.$ base pairs (bp), $\sim 4000$ genes] and roughly one-third the size of the $P$. falciparum genome. Compared to Plasmodium, Theileria appears to have a reduced metabolic capability, with deficiencies in some biosynthetic pathways (e.g. haem and type II fatty acid biosynthesis). Despite high conservation of gene sequences and synteny between $T$. parva and $T$. annulata, several unequally expanded gene families and species-specific genes were revealed [10]. In addition, several candidate regulators of host cell transformation have been proposed $[10,12]$. Similar to many other parasitic protozoa, both Theileria species have tandem arrays of genus-specific hypervariable gene families adjacent to the telomeres. Another highlight of the Theileria genome comparison was the identification of a Theileria-specific highly polymorphic protein domain, known as FAINT (Frequently Associated IN Theileria), that is present in multiple copies (>900 copies of FAINT domains per genome) in a large number of secreted proteins. In the future, extending these comparisons to the genome of a non-transforming Theileria species, currently under way (Sugimoto, C., personal communication), will be of particular value.

Complementing the genome sequences of the two Theileria species are a vast amount of transcriptome data for both. These include a set of over 16500 expressed sequence tag (EST) sequences representing the transcriptome of the macroschizont, merozoite and piroplasm of $T$. annulata, and over a million sequence signatures obtained from the schizont stage of $T$. parva by massively parallel signature sequencing (MPSS). The MPSS method identified and quantified the transcriptional activity of more than $73 \%$ of the predicted genes [13]. Using the T. annulata EST data, evidence of positive immune selection was revealed in several genes expressed at the macroschizont and merozoite stages [10].

The genomes of several Babesia species, such as Babesia bovis, B. bigemina, B. equi (Theileria equi) and B. microti, are at different stages of completion. The genome sequences of $B$. bovis and B. bigemina are now searchable for BLASTbased queries (see Box 2) and the fully annotated genome sequence of $B$. bovis should be publicly available before the end of 2007. This will kick-start the post-genomic era of Babesia research. Recently, analysis of a set of 13000 EST sequences from the erythrocytic stage of $B$. bovis revealed the transcriptional profiles of $\sim 2600$ genes [14].

Once complete genome sequences are available for two or more of the Babesia species mentioned above, largescale comparative genomics analysis is expected to define a significant portion of the repertoire of antigenic variant genes and gene families, such as the variant erythrocyte surface antigen (VESA), and might shed further light on the mechanism of antigenic variation in $B$. bovis [15]. In addition, it will undoubtedly help to identify the metabolic potential and weaknesses of the genus Babesia, spot species-specific differences and define genes that are under diversifying (positive) selection.

The genomes of a large number of tick-borne viruses have been sequenced (Box 2), some of which pose a significant threat to the health of humans or livestock. One of the key aims of comparing the genomes of different tick-borne viral strains is to determine the molecular adaptations that enable them to infect, replicate and survive within ticks and vertebrate cells [16].

\section{Tick genomes and genomic resources}

The sequencing of the genomes of the ticks themselves is lagging, mostly because of the unexpectedly large size of tick genomes. Estimates for three species are currently available, the smallest being $\sim 10^{9}$ bp (Amblyomma americanum) and the largest $\sim 7 \times 10^{9}$ bp [Rhipicephalus (Boophilus) microplus]; the Ixodes scapularis genome is estimated to be $2 \times 10^{9}$ bp [17,18]. Nevertheless, sequencing of the genome of I. scapularis is under way [19]. Pilotscale studies on the genome of $R$. (Boophilus) microplus [20] are progressing and it is expected that this will strengthen a future proposal to sequence the genome more fully. Information on ESTs is available for several tick species, including $R$. (Boophilus) microplus [21], A. americanum [22] and Amblyomma variegatum [23]. Some of these have been used to create a repository of clustered and auto-annotated data in the form of species-specific gene indices (Box 2).

\section{Manipulating gene expression}

The genomics technique that has had the greatest single impact on tick research is RNA interference (RNAi) [24]. Several laboratories, using a range of tick genes and tick species, have successfully used RNAi to transiently suppress the expression of specific tick genes. Suppression can be persistent and act transstadially. For example, injection of double-stranded RNA into engorged R. (Boophilus) microplus females led to the downregulation of mRNA levels in eggs and subsequently in hatched larvae for several weeks [25]. RNAi has been used to study gene function and the role of tick genes in pathogen transmission, and as a screening tool for the identification of potential tick antigens for vaccine development $[26,27]$. It could have a great impact on the annotation of tick genomic information, particularly when the technique is coupled with high-throughput analysis in cultured tick cells. Again, the application of RNAi might help to characterize the effect of tick protective antigens on the transmission of tick-borne pathogens ([27,28]; see also the article by de la Fuente et al., this issue). Meanwhile, the ability to transform tick-borne pathogens is being developed, creating another tool to understand gene function. For example, A. phagocytophilum was recently successfully transformed to express green fluorescent protein (GFP) using the $\mathrm{Hi}$ ma1 transposase system [29].

\section{Tick proteins and proteomics approaches}

The impact of genomics on our knowledge of expressed parasite proteins has barely been felt, yet it is here that genome and gene sequences could have a major, short-term 
impact. Detailed molecular information is available for few proteins from tick-borne pathogens and, relatively, for even fewer tick proteins. As an indication of the current limitations, for 20 of the most abundantly expressed proteins from unfed $R$. (Boophilus) microplus larvae, only one could be identified from a mass fingerprint alone, whereas 18 others were tentatively identified through BLAST searches and the limited existing EST database, that is, using genomic resources [30]. The importance of translated gene or genome sequences is likely to be even greater for less abundant proteins. Probably the moststudied tick proteins are those involved in the control of host haemostasis, thought to be critical to the success of the feeding tick (Maritz-Oliver et al., this issue).

\section{Tick-host-pathogen interface}

Individual genomics tools are being used to piece together elements of our understanding of the tick-host-pathogen interface. Host microarrays are being used to look at the tick-host interaction at a level of complexity that was previously unattainable. No work using tick microarrays has yet been published, but the generation of a microarray to explore, for example, pathogen-tick interactions is just a matter of time. The application of microarrays is further discussed by Jensen and Glass (this issue). Host-vectorpathogen interactions have been studied through the identification of genes differentially expressed in Dermacentor variabilis ovaries in response to rickettsial infection [31] and in salivary glands of female Rhipicephalus appendiculatus infected with $T$. parva [32], whereas a signal sequence trap has been used to identify secreted proteins in salivary glands of $A$. variegatum and $R$. appendiculatus [33]. The association between the tick vector and the pathogen is exemplified by the identification of a tick receptor that is required for $B$. burgdorferi colonization of $I$. scapularis [34]. The application of the tools of genomics to increase our understanding of the complexities of the three-way interaction between I. scapularis, B. burgdorferi and the mammalian host is described by Hovius et al. (this issue). Another example is the Anaplasma-Ixodes interface. Anaplasma probably benefits from anti-inflammatory molecules secreted by the tick into the host, while also using a tick protein to facilitate its establishment in the tick's salivary gland [35].

\section{Genomics and traditional biology}

Genomics tools do not substitute for established approaches to the study of ticks and tick-borne pathogens, but rather complement them. Biological techniques that complement genomics approaches must be made available and must continue to be developed, because future success will depend on this association. Two reviews in this issue describe such techniques. Guerin and Krober summarize recent advances in the development of in vitro systems for the feeding of ticks. These are an essential surrogate biological tool that can potentially circumvent raising and feeding ticks on mammalian hosts, while allowing temporal and physiological aspects of feeding to be studied. Second, several laboratories have established cell lines from different tick species, although this remains a difficult, slow process. Progress in this area and the application of tick cell lines to study tick-pathogen interactions is reviewed by Bell-Sakyi et al. (this issue).

In the area of parasite control, established approaches remain vitally important and capable of great improvement. The current status of live attenuated vaccines against bovine babesiosis, heartwater and tropical theileriosis is summarized by Shkap et al. (this issue).

\section{Genomes and proteins: where to from here?}

Genome sequences of tick-borne pathogens are accumulating steadily. It is inevitable that the number of available sequences will grow, probably at an accelerating rate. The lack of comparable tick genome sequence data is now a barrier to progress. However, this scenario is set to change as new high-throughput sequencing technologies have the potential to bring the cost of sequencing down significantly. The performance of these new-generation DNA sequencing machines (e.g. 454, Solexa) is currently being evaluated by several genome sequencing centres around the world. The size of the genome might no longer be a key limiting factor because of the tremendous output potential of these new technologies.

A complete tick genome sequence would reveal the genetic landscape of ticks in terms of the organization, structure and identity of coding and non-coding DNA, and provide molecular reagents with which to dissect its biology and its interaction with pathogen and host. Outcomes would be as diverse as the resolution of issues in tick phylogeny, the prediction of the components of pathways leading to novel targets for acaricide and vaccine development, and the generation of global transcriptional networks to derive clues to genes with important roles in tick biology or at the tick-vector or tick-pathogen interfaces. Even without whole-genome sequences, useful sequence information can be obtained with less effort and cost. Expansion of the sequencing of expressed genes, preferably full-length sequences, would give us a valuable resource, for example, for the construction of partial microarrays, and key information for any proteomics investigation of ticks.

Current information on tick protein sequences is extremely restricted, limiting the application of proteomics. Homology matches have limited usefulness. We urgently need more information on the complement of proteins expressed in a variety of tick tissues, life stages and species, ideally from more than one species. Sequencing of another tick species within the metastriata should be initiated.

There are likely to be practical outcomes of such research that are unrelated to parasite control. Ticks, for example, are probable sources of novel pharmacological agents, as recent examples such as proteins with both histamine- and serotonin-binding sites [36], haemostatic agents (Maritz et al.., this issue) and C5-specific inhibitors [37] show.

\section{New tools for the control of parasites and disease}

All this work must eventually be aimed at developing new modes of intervention: novel drug targets, new vaccines against ticks and tick-borne diseases, or alternative techniques for control. As an example, ESTs from $R$. (Boophilus) microplus and $R$. appendiculatus were recently used as 
a starting point for the identification of possible anti-ectoparasitic drug targets [38]. Potentially, threedimensional structures can be predicted based on homology, leading to rational drug design. Silencing of potential drug targets in ticks using RNAi would complement this approach.

Genomics information provides a resource that can be mined for antigens that protect against tick infestations and infection by and transmission of tick-borne pathogens. For example, the post-genomics era of Theileria spp. offers an opportunity to develop new vaccines by following 'reverse vaccinology' strategies. Using the genome sequence data on $T$. parva, several promising candidate vaccine antigens that are recognized by bovine $\mathrm{CD}^{+}$cytotoxic $\mathrm{T}$ lymphocytes have been identified [39]. Other high-throughput approaches are under way, such as parasite transcriptome studies using whole-genome microarrays of $T$. parva and $T$. annulata. Combining analysis of the $A$. marginale genome sequence with mass-spectrometry-based protein sequencing and conventional antibody screening enabled the rapid identification of 24 additional antigenic proteins from this organism [40]. It is important also to recognize that anti-tick vaccines can directly and indirectly affect tick-transmitted disease [28,41]. Arguably, our ability to use current genomics information to best effect is limited by our poor understanding of what is necessary for an effective, protective immune response [42], although this will undoubtedly improve.

Turning the new science into practical solutions will present novel commercial challenges. Genomics will lead to control technologies with characteristics different from those of current acaricides and drugs. Vaccines, RNAi and transgenics will typically have greater specificity for a limited range of tick species or a particular disease organism. This has both advantages and disadvantages. Manufacturing costs may be lower and require only generic manufacturing facilities, which are likely to be locally available. Regulatory processes may be variable and of unpredictable stringency. Recombinant vaccines will probably be readily accepted, as several are already on the market, but products delivered via live viral vectors, RNAi or transgenic animals are likely to find that regulatory processes are a significant barrier to their introduction, at least until there is greater familiarity with such novel products. Collectively, these factors - the potential for local manufacture, local regulation and local application - may make the current model of commercial delivery, with its reliance on the resources of global animal health companies, less relevant. Cost-effective control programs in the future will demand both currently available and new technologies derived from genomics studies supported by local expertise and resources.

\section{Acknowledgements}

Formation of genomic consortia on ticks and tick-borne pathogens is facilitated by the Integrated Consortium on Ticks and Tick-borne Diseases (ICTTD-3), financed by the International Cooperation Program of the European Union through Coordination Action Project number 510561 .

\section{References}

1 Jongejan, F. and Uilenberg, G. (2004) The global importance of ticks. Parasitology 129, S3-S14
2 Brayton, K.A. et al. (2005) Complete genome sequencing of Anaplasma marginale reveals that the surface is skewed to two superfamilies of outer membrane proteins. Proc. Natl. Acad. Sci. U. S. A. 102, 844-849

3 Hotopp, J.C. et al. (2006) Comparative genomics of emerging human ehrlichiosis agents. PLoS Genet. 2, e21

4 Collins, N.E. et al. (2005) The genome of the heartwater agent Ehrlichia ruminantium contains multiple tandem repeats of actively variable copy number. Proc. Natl. Acad. Sci. U. S. A. 102, 838-843

5 Frutos, R. et al. (2006) Comparative genomic analysis of three strains of Ehrlichia ruminantium reveals an active process of genome size plasticity. J. Bacteriol. 188, 2533-2542

6 Fraser, C.M. et al. (1997) Genomic sequence of a Lyme disease spirochaete, Borrelia burgdorferi. Nature 390, 580-586

7 Glöckner, G. et al. (2004) Comparative analysis of the Borrelia garinii genome. Nucleic Acids Res. 32, 6038-6046

8 Glöckner, G. et al. (2006) Comparative genome analysis: selection pressure on the Borrelia vls cassettes is essential for infectivity. BMC Genomics 7, 211

9 Ramamoorthi, N. et al. (2005) The Lyme disease agent exploits a tick protein to infect the mammalian host. Nature 436, 573-577

10 Pain, A. et al. (2005) Genome of the host-cell transforming parasite Theileria annulata compared with T. parva. Science 309, 131-133

11 Gardner, M.J. et al. (2005) Genome sequence of Theileria parva, a bovine pathogen that transforms lymphocytes. Science 309, 134-137

12 Shiels, B. et al. (2006) Alteration of host cell phenotype by Theileria annulata and Theileria parva: mining for manipulators in the parasite genomes. Int. J. Parasitol. 36, 9-21

13 Bishop, R. et al. (2005) Analysis of the transcriptome of the protozoan Theileria parva using MPSS reveals that the majority of genes are transcriptionally active in the schizont stage. Nucleic Acids Res. 33, $5503-5511$

14 de Vries, E. et al. (2004) Expressed sequence tag (EST) analysis of the erythrocytic stages of Babesia bovis. Vet. Parasitol. 138, 61-74

15 Allred, D.R. et al. (2000) The ves multigene family of Babesia bovis encodes components of rapid antigenic variation at the infected erythrocyte surface. Mol. Cell 5, 153-162

16 Labuda, M. and Nuttall, P.A. (2004) Tick-borne viruses. Parasitology 129, S221-S245

17 Palmer, M.J. et al. (1994) Genome size and organization in the ixodid tick Amblyomma americanum (L). Insect Mol. Biol. 3, 57-62

18 Ullmann, A.J. et al. (2005) Genome size and organization in the blacklegged tick, Ixodes scapularis and the Southern cattle tick, Boophilus microplus. Insect Mol. Biol. 14, 217-222

19 Hill, C.A. and Wikel, S.K. (2005) The Ixodes scapularis Genome Project: an opportunity for advancing tick research. Trends Parasitol. 21, 151153

20 Guerrero, F.D. et al. (2006) Sequencing a new target genome: the Boophilus microplus (Acari: Ixodidae) genome project. J. Med. Entomol. 43, 9-16

21 Guerrero, F.D. et al. (2005) BmiGI: A database of cDNAs expressed in Boophilus microplus, the tropical/southern cattle tick. Insect Biochem. Mol. Biol. 35, 585-595

22 Hill, C.A. and Gutierrez, J.A. (2000) Analysis of the expressed genome of the lone star tick, Amblyomma americanum (Acari: Ixodidae) using an expressed sequence tag approach. Microb. Comp. Genomics 5, 89102

23 Nene, V. et al. (2002) AvGI, an index of genes transcribed in the salivary glands of the ixodid tick Amblyomma variegatum. Int. J. Parasitol. 32, 1447-1456

24 Aljamali, M.N. et al. (2002) RNA interference: applicability in tick research. Exp. Appl. Acarol. 28, 89-96

25 Nijhof, N.M. et al. (2007) Gene silencing of the tick protective antigens, Bm86, Bm91 and subolesin, in the one-host tick Boophilus microplus by RNA interference. Int. J. Parasitol. 37, 653-662

26 de la Fuente, J. et al. (2005) RNA interference screening in ticks for identification of protective antigens. Parasitol. Res. 96, 137-141

27 de la Fuente, J. and Kocan, K.M. (2006) Strategies for development of vaccines for control of ixodid tick species. Parasite Immunol. 28, 275283

28 Labuda, M. et al. (2006) An antivector vaccine protects against a lethal vector-borne pathogen. PloS Pathog. 2, e27

29 Felsheim, R.F. et al. (2006) Transformation of Anaplasma phagocytophilum. BMC Biotechnol. 6, 42 
30 Untalan, P.M. et al. (2005) Proteome analysis of abundantly expressed proteins from unfed larvae of the cattle tick, Boophilus microplus Insect Biochem. Mol. Biol. 35, 141-151

31 Mulenga, A. et al. (2003) Dynamics of Rickettsia-tick interactions: identification and characterization of differentially expressed mRNAs in uninfected and infected Dermacentor variabilis. Insect Mol. Biol. 12, 185-193

32 Nene, V.et al. (2004) Genes transcribed in the salivary glands of female Rhipicephalus appendiculatus ticks infected with Theileria parva. Insect Biochem. Mol. Biol. 34, 1117-1128

33 Lambson, B. et al. (2005) Identification of candidate sialome components expressed in ixodid tick salivary glands using secretion signal complementation in mammalian cells. Insect Mol. Biol. 14, 403-414

34 Pal, U. et al. (2004) TROSPA, an Ixodes scapularis receptor for Borrelia burgdorferi. Cell 119, 457-468

35 Foley, J. and Nieto, N. (2007) Anaplasma phagocytophilum subverts tick salivary gland proteins. Trends Parasitol. 23, 3-5

36 Sangamnatdej, S. et al. (2002) A high affinity serotonin- and histaminebinding lipocalin from tick saliva. Insect Mol. Biol. 11, 79-86
37 Hepburn, N.J. et al. (2007) In vivo characterisation and therapeutic efficacy of a C5-specific inhibitor from the soft tick Ornithodoros moubata. J. Biol. Chem. 282, 8292-8299

38 Krasky, A. et al. (2007) A combined bioinformatics and chemoinformatics approach for the development of new antiparasitic drugs. Genomics 89, 36-43

39 Graham, S.P. et al. (2006) Theileria parva candidate vaccine antigens recognized by immune bovine cytotoxic T lymphocytes. Proc. Natl. Acad. Sci. U. S. A. 103, 3286-3291

40 Lopez, J.E. et al. (2005) Identification of novel antigenic proteins in a complex Anaplasma marginale outer membrane immunogen by mass spectrometry and genomic mapping. Infect. Immun. 73, 8109-8118

41 Valle, M.R. et al. (2004) Integrated control of Boophilus microplus ticks in Cuba based on vaccination with the anti-tick vaccine Gavac ${ }^{\mathrm{TM}}$. Exp. Appl. Acarol. 34, 375-382

42 Willadsen, P. (2006) Tick control: thoughts on a research agenda. Vet. Parasitol. 138, 161-168

43 Bisby, F.A. (2000) The quiet revolution: biodiversity informatics and the internet. Science 289, 2309-2312

\section{Elsevier.com - linking scientists to new research and thinking}

Designed for scientists' information needs, Elsevier.com is powered by the latest technology with customer-focused navigation and an intuitive architecture for an improved user experience and greater productivity.

The easy-to-use navigational tools and structure connect scientists with vital information - all from one entry point. Users can perform rapid and precise searches with our advanced search functionality, using the FAST technology of Scirus.com, the free science search engine. Users can define their searches by any number of criteria to pinpoint information and resources. Search by a specific author or editor, book publication date, subject area - life sciences, health sciences, physical sciences and social sciences - or by product type. Elsevier's portfolio includes more than 1800 Elsevier journals, 2200 new books every year and a range of innovative electronic products. In addition, tailored content for authors, editors and librarians provides timely news and updates on new products and services.

Elsevier is proud to be a partner with the scientific and medical community. Find out more about

our mission and values at Elsevier.com. Discover how we support the scientific, technical and medical communities worldwide through partnerships with libraries and other publishers, and grant awards from The Elsevier Foundation.

As a world-leading publisher of scientific, technical and health information, Elsevier is dedicated to linking researchers and professionals to the best thinking in their fields. We offer the widest and deepest coverage in a range of media types to enhance cross-pollination of information, breakthroughs in research and discovery, and the sharing and preservation of knowledge.

\section{Elsevier. Building insights. Breaking boundaries. www.elsevier.com}

